\title{
Homogenization in periodically heterogeneous elastic bodies with multiple micro-contact
}

Mathematics and Mechanics of Solids 2014, Vol. 19(8) 1011-1021 (C) The Author(s) 2013 Reprints and permissions: sagepub.co.uk/journalsPermissions.nav DOI: $10.1177 / 1081286513501104$ mms.sagepub.com

\author{
Sebastian Fillep \\ Chair of Applied Mechanics, University Erlangen-Nuremberg, Erlangen, Germany
}

Julia Orlik and Zoufine Bare

Fraunhofer Institut für Techno- und Wirtschaftsmathematik, Kaiserslautern, Germany

\author{
Paul Steinmann \\ Chair of Applied Mechanics, University Erlangen-Nuremberg, Erlangen, Germany
}

Received 10 April 2013; accepted I5 July 2013

\begin{abstract}
The aim of this contribution is to compute the effective in-plane tension and shear behaviour of textile-like elastic materials, that is, plates or shells with a periodic micro-structure composed of long woven or knitted fibres. The knitting or weaving results in multiple periodic contact between fibres. Mathematically the problem can be formulated as an in-plane elasticity problem defined in a heterogeneous domain with $\varepsilon$-periodic micro-structure, including multiple microcontact between the structural components, which is described by the Signorini and Tresca-friction contact conditions. The asymptotic analysis and homogenized limit for such problems was recently obtained by Damlamian et al. via periodic unfolding strategy. These results are briefly recalled in the paper. The obtained two-scale algorithm is implemented for some specific textile materials, which are macroscopic shells with a 3-D microstructure including contact. For each macroscopic deformation state, a contact problem in the periodicity cell or representative volume element is solved and the corresponding non-linear macroscopic stress-strain relation is obtained. The results are illustrated by the simulation of woven and knitted textiles.
\end{abstract}

\section{Keywords}

Contact problems, effective elasto-plasticity, textile structures

\section{Introduction}

The increased application of textiles in various technical fields is decisively influenced by the recent development of different fibre shapes and the improvement of their properties. For example, textiles find application in architecture as construction elements and design material, and as geo-textiles for erosion defence of embankments or as drainage layers. Due to their adjustable porosity they are also well suited as filter material. Their flexibility makes them interesting for usage as carrier material for electronic devices or flexible solar cells. Due to the variety of processable materials, even bio-compatible structures for medical applications (e.g. implants) can be found. 
The consideration of the material behaviour of textiles at different length scales is essential to understanding the behaviour of these heterogeneous materials and to define adequate constitutive laws. Using feasible homogenization techniques permits development of scale transitions between the various length scales involved, whereby the challenge is to transfer information across length scales. Within homogenization-based approaches the macroscopic constitutive behaviour of the inhomogeneous material is modelled by means of an appropriate micro-scale representative volume element (RVE) or periodicity cell.

The quasi-macroscopic textile behaviour is usually non-linear and results mainly from the contact interaction between the fibres, whereby the fibres themselves can be assumed to be linear elastic. There is a known way to linearize the problem and to separate the scales, which will be not considered in this paper. For numerical computations of contact problems, penalization and regularization methods are often used (see e.g. [2, 3]). Hereby, the non-penetration condition can be penalized and added to the variational inequality and the non-differentiable friction functional can be approximated by a differentiable one. Furthermore, if we take into consideration the periodic structure of woven or knitted textiles, large sliding between fibres will be not allowed. Then, according to [2] and [3], the non-linear Signorini and Tresca contact conditions could be replaced by an anisotropic linear Robin condition; see for example [4]. Homogenization for such periodic problems with linear Robin-type boundary conditions on the oscillating interface was considered in [5]. Such problems are completely scaleseparated and the only difference to the homogenization in linear elasticity is the Robin interface-term in the auxiliary problem, which also enters the effective coefficients.

Two-scale asymptotic analysis of multi-scale contact problems was earlier considered in [6-8], the book [9] and recently extended to frictional contact in [1]. We will briefly recall these results in Section 2, which start with the strong and weak formulation of the multi-scale contact problem as well as the corresponding minimization problem and the known existence results for contact problems. Damlamian et al. [1] provide the unfolding-based convergence of the micro-contact problem to an auxiliary contact problem on the periodicity cell, containing the macroscopic deformation as a parameter or internal variable and the homogenized elasto-plastic problem.

Due to the contact phenomena, the quasi-static problem is non-linear. Scale separation could however be possible, even in non-linear problems, if the energy or the stresses (elastic and dissipative ones) are homogeneous functions of a certain order of the strain tensor (symmetric part of the displacement gradient). The general statement of the frictional contact problem does not allow a scale separation. Therefore, homogenization is not applicable in its original sense and the microscopic problem should be recomputed several times for different values of the macroscopic deformation in different directions. Then some response curves for different loading directions (axial tension, shear, etc.) can provide the anisotropic quasi-macroscopic material behaviour. The response curves can then be approximated by some known phenomenological elasto-plastic models like in [10] and [11].

We illustrate the obtained algorithm by an application to fabric materials. Examples are taken from real industrial applications and are computed by the finite element method also involving a contact formulation.

However, we need to note here that Tresca friction is just an approximation (in time) of the Coulomb law and can be stated only on the known contact interface, in other words, on each incremental quasi-static step; see [12].

\section{Multi-scale contact problem: Statement, modelling and assumptions}

We denote by $Y$ a unit periodicity cell and by $0<\varepsilon \ll 1$ a scaling parameter, and consider an $\varepsilon Y$-periodic structure in $\mathbb{R}^{n}$ composed of a homogeneous solid material with periodically distributed cracks or consisting of long fibres being in multiple periodic contact with each other.

We define a domain $\Omega^{\varepsilon}$ as an intersection of the periodic structure with a finite Lipschitz domain $\Omega \subset \mathbb{R}^{n}$ and denote its external boundary by $\partial \Omega^{\varepsilon}$, which is decomposed in two parts $\partial \Omega^{\varepsilon}=\partial \Omega_{D}^{\varepsilon} \cup \partial \Omega_{N}^{\varepsilon}$, on which Dirichlet and Neumann boundary conditions are imposed respectively, see Figure 1. We assume that $\partial \Omega^{\varepsilon}$ is a Lipschitz boundary and that each long fibre touches $\partial \Omega$. Furthermore, we denote the intersection of the complete contact boundary with $\Omega$ by $S^{\varepsilon}$, which also is Lipschitz continuous (in the case of cracks, consisting of parts of Lipschitz continuous curves).

Finally, we assume that all domains consist of isotropic elastic materials.

For the displacement vector $\boldsymbol{u}^{\varepsilon}$ and the symmetric fourth-order tensor of elasticity coefficients $a_{i j k l}^{\varepsilon}(\boldsymbol{x})=$ $a_{i j k l}(\boldsymbol{x} / \varepsilon)$, we consider the contact problem consisting of the following: 


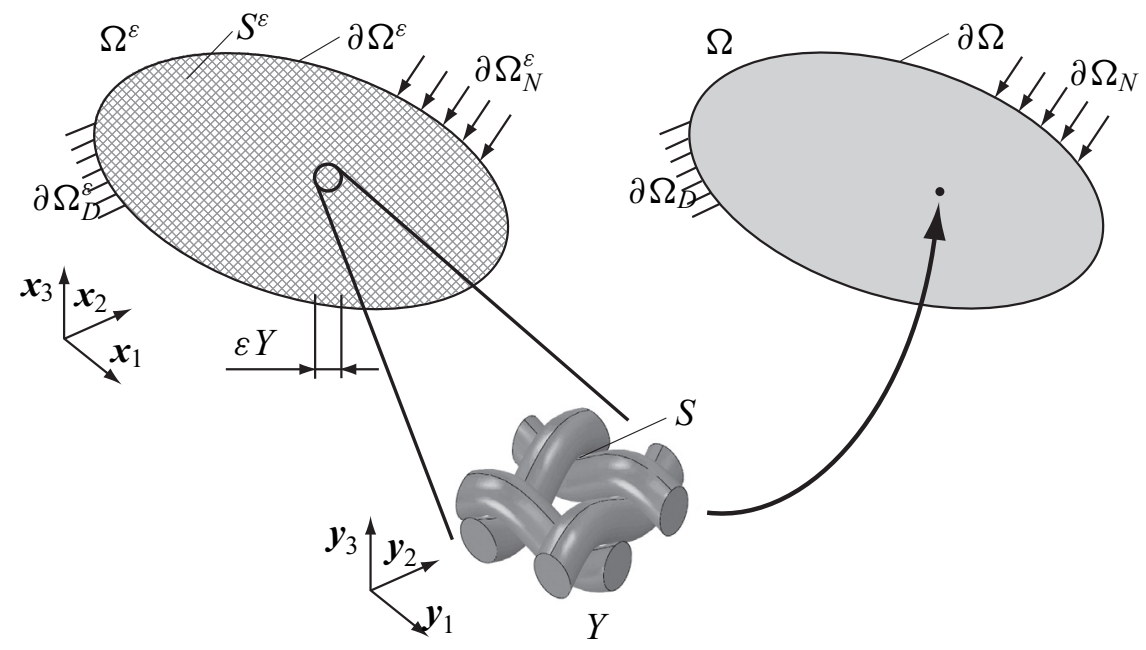

Figure I. Domain $\Omega^{\varepsilon}$ with periodic microstructure including contact.

1. Equilibrium equations:

$$
\begin{aligned}
& -\frac{\partial \sigma_{i j}^{\varepsilon}(\boldsymbol{x})}{\partial x_{j}}=f_{i}^{\varepsilon}(\boldsymbol{x}), \quad \sigma_{i j}^{\varepsilon}(\boldsymbol{x})=a_{i j k l}^{\varepsilon}(\boldsymbol{x}) e_{k l}^{\varepsilon}(\boldsymbol{x}), \\
& e_{k l}^{\varepsilon}=e_{k l}\left(\boldsymbol{u}^{\varepsilon}(\boldsymbol{x})\right)=\frac{1}{2}\left(\frac{\partial u_{k}^{\varepsilon}(\boldsymbol{x})}{\partial x_{l}}+\frac{\partial u_{l}^{\varepsilon}(\boldsymbol{x})}{\partial x_{k}}\right), \\
& \boldsymbol{x} \in \Omega^{\varepsilon} \backslash S^{\varepsilon}
\end{aligned}
$$

2. Conditions on the contact interface:

(i) Non-penetration (Signorini) conditions in the normal direction:

$$
[u]_{\mathrm{n}}^{\varepsilon}(\boldsymbol{x}) \leq g^{\varepsilon}(\boldsymbol{x}), \quad \sigma_{\mathrm{n}}^{\varepsilon}(\boldsymbol{x}) \leq 0, \quad \sigma_{\mathrm{n}}^{\varepsilon}(\boldsymbol{x})\left([u]_{\mathrm{n}}^{\varepsilon}(\boldsymbol{x})-g^{\varepsilon}(\boldsymbol{x})\right)=0 \quad \boldsymbol{x} \in S^{\varepsilon}
$$

(ii) Frictional stick and slip conditions in the tangential surface:

$$
\begin{array}{ll}
\left|\boldsymbol{\sigma}_{\mathrm{t}}^{\varepsilon}(\boldsymbol{x})\right|<G^{\varepsilon}(\boldsymbol{x}), & \Rightarrow \quad[\boldsymbol{u}]_{\mathrm{t}}^{\varepsilon}=0, \quad \boldsymbol{x} \in S^{\varepsilon}, \\
\left|\boldsymbol{\sigma}_{\mathrm{t}}^{\varepsilon}(\boldsymbol{x})\right|=G^{\varepsilon}(\boldsymbol{x}), \quad \Rightarrow \quad \exists \lambda^{\varepsilon} \geq 0 \text { such that }[\boldsymbol{u}]_{\mathrm{t}}^{\varepsilon}=-\lambda^{\varepsilon} \boldsymbol{\sigma}_{\mathrm{t}}^{\varepsilon}, \boldsymbol{x} \in S^{\varepsilon}
\end{array}
$$

(iii) Boundary conditions:

$$
\sigma_{i j}^{\varepsilon} n_{j}^{\varepsilon}(\boldsymbol{x})=t_{i}^{\varepsilon}(\boldsymbol{x}) \quad \boldsymbol{x} \in \partial \Omega_{N}^{\varepsilon} ; \quad u_{i}^{\varepsilon}(\boldsymbol{x})=g_{i 0}(\boldsymbol{x}), \quad \boldsymbol{x} \in \partial \Omega_{D}^{\varepsilon},
$$

where $[\varphi]=\left.\left.\varphi\right|_{S_{+}^{\varepsilon}} ^{\varepsilon} \varphi\right|_{S_{-}^{\varepsilon}},\left(u_{i}^{\varepsilon}\right)_{i=1, \ldots, n}$ is the displacement vector, $\left(e_{i j}^{\varepsilon}\right)_{i, j=1, \ldots, n}$ is the strain tensor (symmetric part of $\nabla \boldsymbol{u}),\left(\sigma_{i j}^{\varepsilon}\right)_{i, j=1, \ldots, n}$ is the Cauchy stress tensor, $g^{\varepsilon}(\boldsymbol{x})$ is the initial gap function between contacting surfaces, $G^{\varepsilon}(\boldsymbol{x})$ is the Tresca friction traction, $\varepsilon$ is a small parameter denoting the periodicity of the microstructure, $\left(f_{i}^{\varepsilon}\right)_{i=1, . ., n}$ is the vector of volume forces, $\left(t_{i}^{\varepsilon}\right)_{i=1, . ., n}$ is the vector of boundary tractions, $\left(g_{i 0}\right)_{i=1, \ldots, n}$ is the vector of boundary displacements, $u_{n}^{\varepsilon}(\boldsymbol{x})=\boldsymbol{u}^{\varepsilon}(\boldsymbol{x}) \cdot \boldsymbol{n}^{\varepsilon}(\boldsymbol{x}), \boldsymbol{u}_{\tau}^{\varepsilon}(\boldsymbol{x})=\boldsymbol{u}^{\varepsilon}(\boldsymbol{x})-u_{n}^{\varepsilon} \boldsymbol{n}^{\varepsilon}(\boldsymbol{x}), \sigma_{n}^{\varepsilon}(\boldsymbol{x})=\left(\boldsymbol{\sigma}^{\varepsilon}(\boldsymbol{x}) \cdot \boldsymbol{n}^{\varepsilon}(\boldsymbol{x})\right) \cdot \boldsymbol{n}^{\varepsilon}(\boldsymbol{x}), \boldsymbol{\sigma}_{\tau}^{\varepsilon}(\boldsymbol{x})=$ $\boldsymbol{\sigma}^{\varepsilon}(\boldsymbol{x}) \cdot \boldsymbol{n}^{\varepsilon}(\boldsymbol{x})-\sigma_{n}^{\varepsilon} \boldsymbol{n}^{\varepsilon}(\boldsymbol{x})$ and $\boldsymbol{n}^{\varepsilon}$ is the unit outward normal vector to $\partial \Omega^{\varepsilon}$. Further, $\left(a_{i h j k}^{\varepsilon}(\boldsymbol{x})\right), i, j, k, h=1, \ldots, n$, is the elasticity tensor, which is major and minor symmetric at each point $\boldsymbol{x} \in \Omega^{\varepsilon}$,

$$
a_{i h j k}^{\varepsilon}(\boldsymbol{x})=a_{j k i h}^{\varepsilon}(\boldsymbol{x})=a_{h i j k}^{\varepsilon}(\boldsymbol{x})=a_{i h k j}^{\varepsilon}(\boldsymbol{x}),
$$

and positive-definite, with elements bounded at each point $\boldsymbol{x} \in \Omega^{\varepsilon}$,

$$
c_{0} \eta_{j k} \eta_{j k} \leq a_{i h j k}^{\varepsilon}(\boldsymbol{x}) \eta_{i h} \eta_{j k} \leq C_{0} \eta_{j k} \eta_{j k}, \text { for all } \eta_{j k}=\eta_{k j} \in \mathbb{R},
$$


where the constants $0<c_{0} \leq C_{0}<\infty$ are independent of $\boldsymbol{x}$. Furthermore, $a_{i h j k}^{\varepsilon}(\boldsymbol{x})=a_{i h j k}(\boldsymbol{x} / \varepsilon), \forall i, j, k, h=$ $1, \ldots n$.

The friction functional defining the dissipation associated with frictional sliding is usually written as

$$
j_{t}^{\varepsilon}(\boldsymbol{v}):=\int_{S^{\varepsilon}} G^{\varepsilon}(\boldsymbol{x})\left|[\boldsymbol{v}]_{\tau}(\boldsymbol{x})\right| d s
$$

(see e.g. $[2,3])$. The solution of the contact problem satisfies the variational inequality

$$
\begin{aligned}
& a\left(\boldsymbol{u}^{\varepsilon}, \boldsymbol{v}^{\varepsilon}-\boldsymbol{u}^{\varepsilon}\right)+j_{t}^{\varepsilon}\left(\boldsymbol{v}^{\varepsilon}\right)-j_{t}^{\varepsilon}\left(\boldsymbol{u}^{\varepsilon}\right) \\
& \quad \geq \int_{\Omega^{\varepsilon} \backslash S^{\varepsilon}} \boldsymbol{f}^{\mathcal{E}}(\boldsymbol{x})\left(\boldsymbol{v}^{\varepsilon}(\boldsymbol{x})-\boldsymbol{u}^{\varepsilon}(\boldsymbol{x})\right) d x+\int_{\partial \Omega_{N}^{\varepsilon}} \boldsymbol{t}^{\varepsilon}(\boldsymbol{x})\left(\boldsymbol{v}^{\varepsilon}(\boldsymbol{x})-\boldsymbol{u}^{\varepsilon}(\boldsymbol{x})\right) d s .
\end{aligned}
$$

for all test functions from the cone $\mathcal{K}^{\varepsilon}:=\left\{\boldsymbol{v}^{\varepsilon} \in \boldsymbol{H}^{1}\left(\Omega^{\varepsilon} \backslash S^{\varepsilon}\right) \mid \boldsymbol{v}^{\varepsilon}=\boldsymbol{g}_{0}(\boldsymbol{x})\right.$ on $\partial \Omega_{D}^{\varepsilon}, \quad[v]_{n}^{\varepsilon} \leq g^{\varepsilon}$ on $\left.S^{\varepsilon}\right\}$. Here, we introduced a new notation for the bilinear form

$$
a\left(\boldsymbol{u}^{\varepsilon}, \boldsymbol{v}^{\varepsilon}\right):=\int_{\Omega^{\varepsilon} \backslash S^{\varepsilon}} \boldsymbol{a}^{\varepsilon} \boldsymbol{e}\left(\boldsymbol{u}^{\varepsilon}\right) \boldsymbol{e}\left(\boldsymbol{v}^{\varepsilon}\right) d x=\int_{\Omega^{\varepsilon} \backslash S^{\varepsilon}} a_{i j k l}^{\varepsilon} \frac{\partial u_{k}^{\varepsilon}(\boldsymbol{x})}{\partial x_{l}} \frac{\partial v_{i}^{\varepsilon}(\boldsymbol{x})}{\partial x_{j}} d x
$$

Assumption 1 (i) We suppose the initial gap to be of the size of the periodicity cell, in other words,

$$
g^{\varepsilon}(\boldsymbol{x})=\varepsilon \bar{g}\left(\frac{\boldsymbol{x}}{\varepsilon}\right) .
$$

(ii) The friction traction value is simply a material characteristic, in other words,

$$
G^{\varepsilon}(\boldsymbol{x})=G\left(\frac{\boldsymbol{x}}{\varepsilon}\right) \geq 0 \text { on } S^{\varepsilon} .
$$

The existence of the solution to the contact problem (7) for a fixed $\varepsilon$ is a well-studied subject (see Theorem 2.2 in [13] or Theorem 5.1 in [14]). Since the tensor of elastic coefficients is symmetric and the function under the interface integral $(|\cdot|)$ is convex, problem (7) is equivalent to the minimization of the functional

$$
\begin{aligned}
& I^{\varepsilon}\left(\boldsymbol{v}^{\varepsilon}\right)=\frac{1}{2} a\left(\boldsymbol{v}^{\varepsilon}, \boldsymbol{v}^{\varepsilon}\right)+j_{t}^{\varepsilon}\left(\boldsymbol{v}^{\varepsilon}\right) \\
& -\int_{\Omega^{\varepsilon} \backslash S^{\varepsilon}} \boldsymbol{f}^{\mathcal{E}}(\boldsymbol{x})\left(\boldsymbol{v}^{\varepsilon}(\boldsymbol{x})\right) d x+\int_{\partial \Omega_{N}^{\varepsilon}} \boldsymbol{t}^{\varepsilon}(\boldsymbol{x})\left(\boldsymbol{v}^{\varepsilon}(\boldsymbol{x})\right) d s
\end{aligned}
$$

on the set of $\boldsymbol{v}^{\varepsilon} \in \mathcal{K}^{\varepsilon}$.

The proof of the theorem is based on the fact that we have a sum of two functionals. The first one is convex differentiable and the second one is convex lower semicontinuous. The first one, the bilinear form, grows as $O\left(\left\|v^{\varepsilon}\right\|^{2}\right)$ and the second, $j_{t}^{\varepsilon}$, as $O\left(\left\|v^{\varepsilon}\right\|\right)$ when $\left\|v^{\varepsilon}\right\| \rightarrow \infty$. That proves the coerciveness of functional (8).

\section{Homogenization: Result from analysis}

The compactness and convergence of the solution to the multi-scale contact problem for a structure with open and closed cracks was recently investigated in [1]. The result can also be applied to long-fibre materials and is given by the following proposition.

Proposition 1.(Homogenized and corrector (RVE) problems) When $\varepsilon \rightarrow 0$, the sequence $\boldsymbol{u}^{\varepsilon}$ of solutions to (1)-(3) has a subsequence strongly convergent to $\boldsymbol{u}^{0}(\boldsymbol{x})$, and the sequence $\boldsymbol{e}^{\varepsilon}(\boldsymbol{x})$ has a subsequence two-scale convergent to $\boldsymbol{e}\left(\boldsymbol{u}^{0}(\boldsymbol{x})\right)+\boldsymbol{e}_{y}\left(\boldsymbol{u}^{1}(\boldsymbol{x}, \boldsymbol{y})\right)$ (the symmetric part of $\left.\nabla \boldsymbol{u}^{0}(\boldsymbol{x})+\nabla_{y} \boldsymbol{u}^{1}(\boldsymbol{x}, \boldsymbol{y})\right)$, where $\left(\boldsymbol{u}^{0}, \boldsymbol{u}^{1}\right) \in \boldsymbol{H}^{1}\left(\Omega, \partial \Omega_{D}\right) \times$ $\boldsymbol{L}^{2}(\Omega, \mathcal{K})$ is a solution of the two-scale homogenized system, with $\lim _{\varepsilon \rightarrow 0}$ in (7). 
(i) Auxiliary contact problem in the periodicity cell for the corrector $\boldsymbol{u}^{1}$

for some given $\boldsymbol{u}^{0}$ :

$$
\begin{array}{r}
\int_{Y} \boldsymbol{a}\left(\boldsymbol{e}\left(\boldsymbol{u}^{0}\right)+\boldsymbol{e}_{y}\left(\boldsymbol{u}^{1}\right)\right)\left(\boldsymbol{e}_{y}\left(\boldsymbol{v}^{1}\right)-\boldsymbol{e}_{y}\left(\boldsymbol{u}^{1}\right)\right) d y \\
+\int_{S} G(\boldsymbol{y})\left(\left|\left[\boldsymbol{v}^{1}\right]_{\tau}(\boldsymbol{x}, \boldsymbol{y})\right|-\left|\left[\boldsymbol{u}^{1}\right]_{\tau}(\boldsymbol{x}, \boldsymbol{y})\right|\right) d s_{y} \geq 0,
\end{array}
$$

for any $\boldsymbol{v}^{1} \in \mathcal{K}$, where the cone $\mathcal{K}:=\left\{\boldsymbol{v}^{1} \in \boldsymbol{L}^{2}\left(\Omega, \boldsymbol{H}_{\text {per }}^{1}(Y \backslash S)\right) \mid[v]_{n}^{1}(\boldsymbol{x}, \boldsymbol{y}) \leq \bar{g}(\boldsymbol{y})\right.$ for $\boldsymbol{y} \in S$, a.e. $\left.\boldsymbol{x} \in \Omega\right\}$.

(ii) Homogenized problem for $\boldsymbol{u}^{0}$ :

$$
\int_{\Omega} \boldsymbol{\sigma}^{h o m}\left(\boldsymbol{x}, \boldsymbol{e}\left(\boldsymbol{u}^{0}\right)\right) \boldsymbol{e}\left(\boldsymbol{v}^{0}\right) d x=\int_{\Omega} \boldsymbol{f}(\boldsymbol{x}) \boldsymbol{v}^{0}(\boldsymbol{x}) d x+\int_{\partial \Omega_{N}} \boldsymbol{t}(\boldsymbol{x}) \boldsymbol{v}^{0}(\boldsymbol{x}) d s_{x},
$$

for any $\boldsymbol{v}^{0} \in \boldsymbol{H}^{1}\left(\Omega, \partial \Omega_{D}\right)$, where $\boldsymbol{H}^{1}\left(\Omega, \partial \Omega_{D}\right):=\left\{\boldsymbol{v} \in \boldsymbol{H}^{1}(\Omega) \mid v_{n}=g_{0}(\boldsymbol{x})\right.$ on $\left.\partial \Omega_{D}\right\}$ and

$$
\boldsymbol{\sigma}^{h o m}\left(\boldsymbol{x}, \boldsymbol{e}\left(\boldsymbol{u}^{0}\right)\right) \doteq \frac{1}{|Y|} \int_{Y} \boldsymbol{a}(\boldsymbol{x}, \boldsymbol{y})\left(\boldsymbol{e}\left(\boldsymbol{u}^{0}\right)+\boldsymbol{e}_{y}\left(\boldsymbol{u}^{1}\right)\right) d y .
$$

Or in the strong formulation,

$$
\left\{\begin{array}{l}
\boldsymbol{u}^{0} \in \boldsymbol{H}^{1}\left(\Omega, \partial \Omega_{D}\right), \\
-\operatorname{div} \boldsymbol{\sigma}^{\text {hom }}\left(\boldsymbol{x}, \boldsymbol{e}\left(\boldsymbol{u}^{0}\right)\right)=\boldsymbol{f} \text { in } \Omega, \\
\boldsymbol{\sigma}^{\text {hom }} \cdot \boldsymbol{n}=\boldsymbol{t} \quad \text { on } \partial \Omega_{N},
\end{array}\right.
$$

where $\boldsymbol{e}$ denotes the strain tensor.

The proof is given in [1] based on the periodic unfolding strategy recently developed in $[15,16]$. The unfolding method transforms functions defined on varying domains to functions on fixed domains and provides an easy way for proving weak convergence. First, it was proved that the interface jumps of displacement on the contact interface converge to the interface jumps of the first-order correctors (cell or RVE solutions). Subsequently the convergence of the frictional surface integrals was justified, using the first result and the fact that Tresca friction is the continuous convex function of the jump of tangential displacements.

Since the contact problem is non-linear (due to the unknown contact interface and the non-linear friction functional), the complete separation of length scales is impossible.

The limiting problem (11) can be associated with some elasto-plasticity at the macro-scale: the frictional micro-sliding on the oscillating interface results in some remaining deformations, which can be interpreted as plastic strain and should be determined by solving the local contact elasticity problem (9) on the standard periodicity cell, in other words, on the RVE.

\section{Strong formulation of the micro-scale (corrector) problem on the representative volume element}

The heterogeneous material within the periodicity cell or rather the RVE is considered to be a first-order continuum, where the deformation is described by the linearized strain $\boldsymbol{e}$.

We further assume our multi-scale domain $\Omega^{\varepsilon}$ is occupied by the composite solid in the form of a woven layer. This motivates prescribing homogeneous Neumann boundary conditions, that is, applying zero traction conditions on the top and bottom faces of the RVE. This condition is relevant for plates or shells that are not loaded in the out-of-plane direction and leaves the thickness strain unassigned. These boundary conditions in the auxiliary RVE problem (9), formulated to compute the homogenized in-plane constitutive relation (10), are justified by the results from Chapter 3 of [17] on homogenization of linear elasticity equations considered in thin plate domains with in-plane periodic heterogeneities.

The other two pairs of boundary surfaces represent the Dirichlet boundary, where periodic boundary conditions are applied that transfer the macroscopic deformation to the micro level. 
We further assume that the fibres have smooth boundaries and can then rewrite the auxiliary periodic contact elasticity problem (9) for the RVE in strong formulation as follows:

For given $\boldsymbol{u}^{0}$ and fixed $\boldsymbol{x} \in \Omega$,

1. Equilibrium equations:

$$
\operatorname{div}_{y} \sigma\left(y, x, u^{0}\right)=0, \quad \sigma\left(y, x, u^{0}\right)=a(y)\left(e\left(x, u^{0}\right)+e_{y}\left(y, u^{1}\right)\right), \quad y \in Y \backslash S
$$

2. Contact conditions:

$$
\begin{array}{ll}
{[u]_{\mathrm{n}}^{1}(\boldsymbol{y}) \leq \bar{g}(\boldsymbol{y}),} & \sigma_{\mathrm{n}}^{1}(\boldsymbol{y}) \leq 0, \quad \sigma_{\mathrm{n}}^{1}(\boldsymbol{y})\left([u]_{\mathrm{n}}^{1}(\boldsymbol{y})-\bar{g}(\boldsymbol{y})\right)=0 \quad \boldsymbol{y} \in S, \\
\left|\sigma_{\mathrm{t}}(\boldsymbol{y})\right|<G(\boldsymbol{y}), & \Rightarrow \quad[\boldsymbol{u}]_{\mathrm{t}}^{1}=\mathbf{0}, \quad \boldsymbol{y} \in S, \\
\left|\sigma_{\mathrm{t}}(\boldsymbol{y})\right|=G(\boldsymbol{y}), \quad \Rightarrow \quad \exists \lambda \geq 0 \text { such that }[\boldsymbol{u}]_{\mathrm{t}}^{1}=-\lambda \boldsymbol{\sigma}_{\mathrm{t}}, \boldsymbol{y} \in S
\end{array}
$$

3. Boundary conditions:

$$
\begin{aligned}
& \boldsymbol{\sigma} \cdot \boldsymbol{n}=\mathbf{0} \quad \text { on faces } \partial Y \text { of the RVE with the out-of-plane normal } \\
& \boldsymbol{u}^{1} \text { is periodic on opposite faces } \partial Y \text { of the RVE with the in-plane normal, that is, } \\
& \int_{Y} \boldsymbol{u}^{1} d y=0 \quad \text { in-plane }
\end{aligned}
$$

Note that $\boldsymbol{\sigma}^{1} \cdot \boldsymbol{n}$ is then antiperiodic on opposite faces $\partial Y$ of the RVE with the in-plane normal. We assume further that the material behaviour of each micro-structural fibre $\Omega^{j}$ is described by a linear constitutive law,

$$
\boldsymbol{\sigma}(\boldsymbol{y})=\boldsymbol{a}^{j}(\boldsymbol{y}) \boldsymbol{e}_{y}(\boldsymbol{y}) \text {, if } \boldsymbol{y} \in \Omega^{j} .
$$

The contact problem (12)-(14) on the RVE is of the same kind as the multi-scale contact problem, however, the macroscopic strain acts on the RVE as an external loading and the boundary conditions are replaced by the in-plane periodicity conditions for the RVE displacements.

\section{Numerical example}

To evaluate the introduced homogenization method and its finite element implementation the macroscopic homogenized stresses are analysed in the following example. A textile micro-sample is considered and evaluated for different macroscopic deformations. For simplicity, the constitutive behaviour of the fibres is modelled as linear elastic and isotropic, defined by the following constitutive equation:

$$
\boldsymbol{\sigma}=\frac{E}{1+v}\left[\frac{v}{1-2 v} \operatorname{tr}(\boldsymbol{e}) \boldsymbol{I}+\boldsymbol{e}\right]
$$

where $\operatorname{tr}(\boldsymbol{e})$ denotes the trace of the strain tensor; $\boldsymbol{I}$, the identity matrix; E, Young's modulus; and $v$, Poisson's ratio. The material parameters used are characteristic for the elastic behaviour of polymers, for example, polyamide with $E=2000 \mathrm{~N} / \mathrm{mm}^{2}$ and $v=0.33$. Between the fibres contact occurs that leads to non-linearities in the homogenized material behaviour. The normal contact condition is enforced exactly, in contrast to the tangential contact, which is treated by a penalty method. For the relation of the normal and the tangential traction a friction coefficient of $\mu=0.8$ is introduced.

Remark 2. The spacial convergence results and the homogenization algorithm were obtained based on the assumption of Tresca friction. However, Tresca friction is an approximation (with respect to time) of the Coulomb law $G\left(t_{i}\right)=-\mu \sigma_{n}\left(t_{i-1}\right)$ and can be stated only on the known contact interface; that is, on each incremental step (see [12]).

The microscopic mesh is composed of hexahedral full integration elements with quadratic interpolation. 


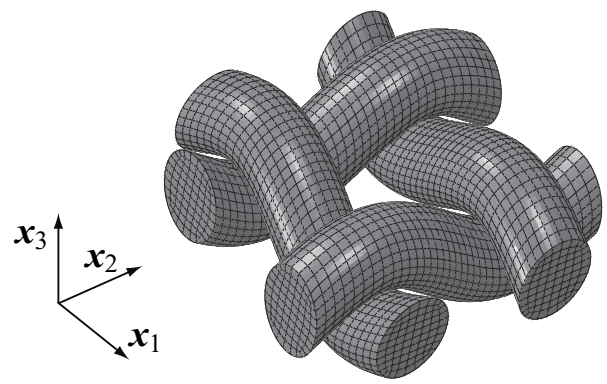

Figure 2. The discretized woven representative volume element.

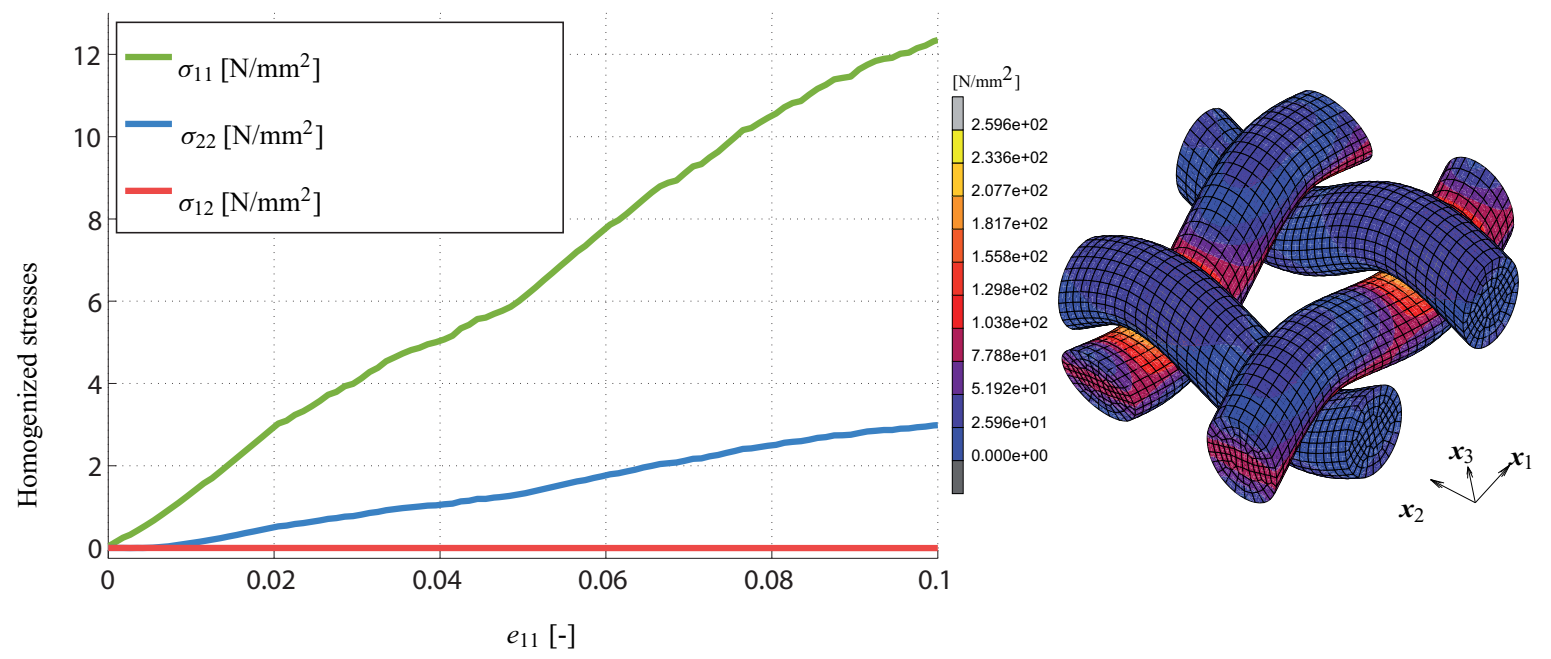

Figure 3. Macroscopic homogenized stress response for stretch deformation (left) and von Mises stress plot of the deformed structure for $e_{11}=0.1$ (right).

\section{I. Effective in-plane properties of a woven plate}

For a woven textile structure a representative unit cell is determined, which has a prismatic shape with the dimensions $4 \times 4 \times 2 \mathrm{~mm}$. To represent the fibres, ideal cylindrical monofilaments are assumed with a fibre diameter of $0.98 \mathrm{~mm}$ (see Figure 2). The microscopic mesh is composed of 10,250 elements. Two typical deformations of the textile are considered. The first is tension in the $\boldsymbol{x}_{1}$ direction as depicted in Figure 3; the second deformation is shear in the textile $\boldsymbol{x}_{1}, \boldsymbol{x}_{2}$-plane as depicted in Figure 4.

The stretch in thickness direction stays undetermined so that the plane stress assumption holds.

The diagram in Figure 3 displays the appearing macroscopic homogenized stresses $\sigma_{11}, \sigma_{22}$ and $\sigma_{12}$ over the stretch $e_{11}$, and the contour plot shows the von Mises stress. Through the elongation of the $\boldsymbol{x}_{1}$-oriented fibres and the resulting flattening of the woven structure, the crossing fibres are pressed on each other. As a result a contact area arises, where stresses are transferred across the fibres. Further, due to the deformation, tangential contact forces appear that lead to a torsion moment within the $\boldsymbol{x}_{2}$-oriented fibres in the section between the contact zones and lead to an increase in the stiffness of the textile structure. By reaching a certain deformation the tangential contact stress limit $G$ is reached so that slip in the contact area occurs. As a consequence the stiffness of the RVE is reduced. The characteristic of the stress $\sigma_{11}$ is quite linear, which results from the linear material behaviour of the $\boldsymbol{x}_{1}$ fibres that contribute to the main part of the stiffness. Some non-linearities are superposed, resulting from the described effect.

In the second example of the woven RVE, shear deformation in the $\boldsymbol{x}_{1}, \boldsymbol{x}_{2}$-plane leads to a rotation and shear dislocation of the fibres around $\boldsymbol{x}_{3}$. In the interaction zones slip occurs and the contact area increases with the rising shear deformation, so that a locking of the structure results in non-linear RVE behaviour. The contact zone is depicted in Figure 5, where, for illustration of the contact zone, a fibre is faded out. Because of these contact conditions the fibres get bent into an S-shape. This S-shape bending is counteracted by a micro-rotation of the initially S-shaped fibres around their mid-axis against the direction of loading. Through the relative movement, 

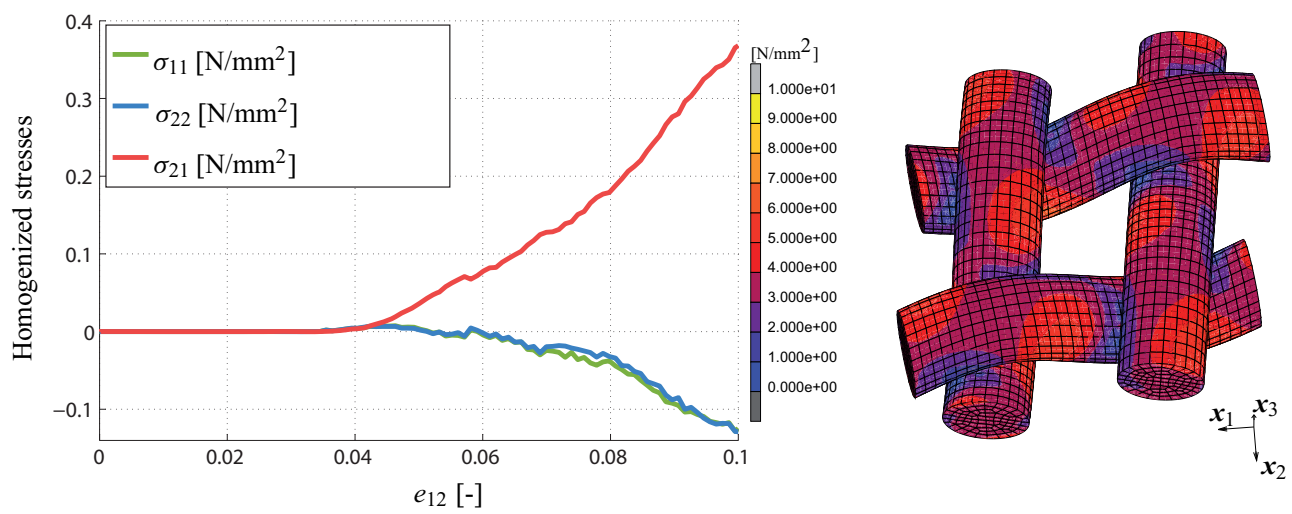

Figure 4. Macroscopic homogenized stress response for shear deformation (left) and von Mises stress plot of the deformed structure for $e_{12}=0.1$ (right).

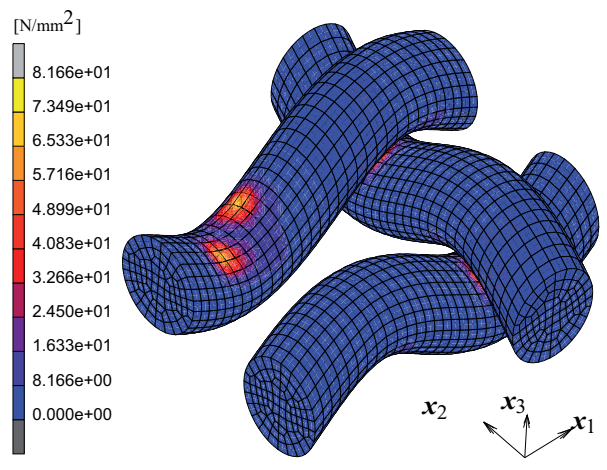

Figure 5. Plot of the contact zone of the deformed structure for $e_{12}=0.1$.

slip in the contact zone occurs, which decreases the stiffness of the structure because the tangential contact stresses are reduced. The expected symmetric properties of the micro-sample can be seen in the approximate equality of the normal stresses $\sigma_{11}=\sigma_{22}$. For deformations $e_{12}<0.04$ no contact area between the fibres arises so the stiffness of the RVE is equal to zero. Thereafter the structure shows a progressive stiffness characteristic resulting from the progressive increasing contact areas.

Comparing the two deformation modes, it is interesting to note that the woven fabric is stiff in normal direction but flexible when shear deformation appears. In both cases non-linear material behaviour occurs, which results from the contact interaction between the fibres.

\subsection{Effective in-plane properties of a knitted plate}

In the second example a prismatic cutout of a knitted textile structure with the dimensions $6.8 \times 8.0 \times 4.2$ $\mathrm{mm}$ is determined as the representative micro-sample. To represent the fibres, ideal cylindrical monofilaments are assumed with a fibre diameter of $1.25 \mathrm{~mm}$ as depicted in Figure 6 . The microscopic mesh is composed of about 7100 elements. For this RVE stretch and shear deformation are also considered and the macroscopic homogenized stresses are analysed. The first is tension in the $\boldsymbol{x}_{1}$ direction as depicted in Figure 7 . The stretch in thickness direction stays undetermined so that the plane stress assumption holds.

The diagram in Figure 8 displays the resulting macroscopic homogenized stresses $\sigma_{11}, \sigma_{22}$ and $\sigma_{12}$ over the stretch $e_{11}$ and the contour plot shows the von Mises stress. The stiffness characteristic of the RVE shows slightly progressive behaviour up to a deformation of about $e_{11}=0.1$ resulting from the increasing point-shaped contact zone. Thereafter the bow fibre enlaces the two other fibre parts which leads to a rapidly increasing contact zone. This results in strong progressive behaviour with a slightly linear continuation. This appearance of the contact zone is depicted in Figure 7 below. In Figure 8(a) the transition from the point-shaped contact zone to the area-shaped contact zone is shown. Figures 8(b) and (c) show the extended two-dimensional contact 


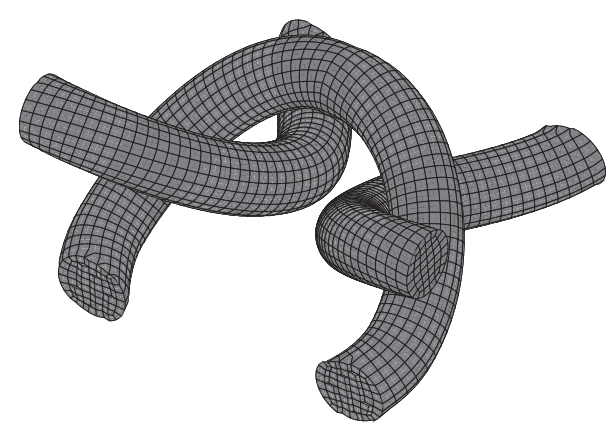

Figure 6. The discretized knitted representative volume element.
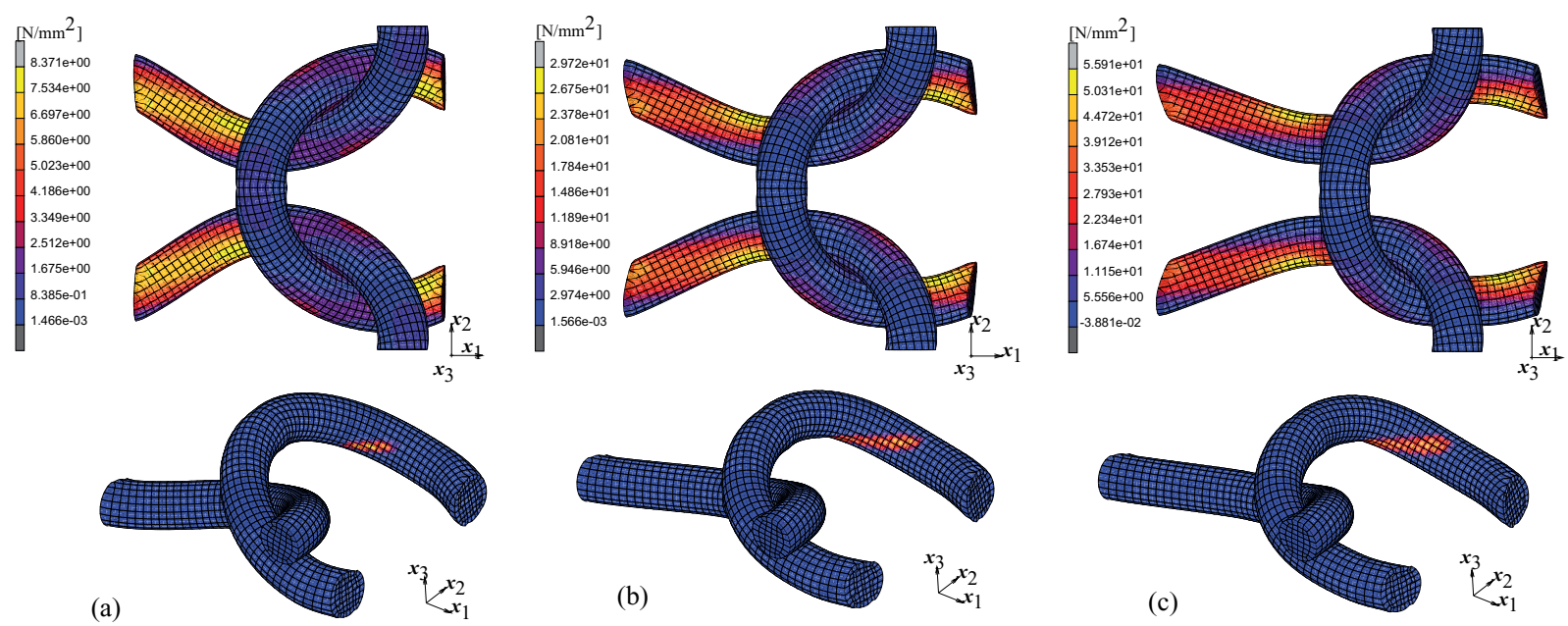

Figure 7. Von Mises plot of the representative volume element for stretch deformation (top) and display of the contact zone (below) for $e_{11}$ of (a) 0.12 , (b) 0.30 , (c) 0.40 .

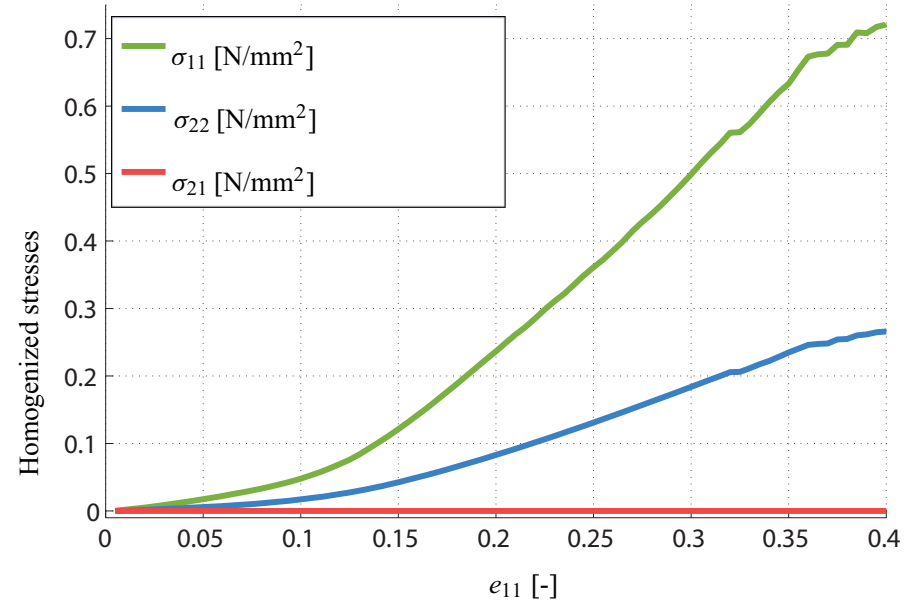

Figure 8. Macroscopic homogenized response of the knitted representative volume element for stretch deformation.

areas which result from the enlacing of the fibres. Beyond a deformation of $e_{11}=0.35$ the characteristic is more discontinuous. A reason for this is the alternating tension of the fibres and the slipping in the fibre bow.

In the second example for the knitted RVE, shear deformation $e_{12}$ in the textile $\boldsymbol{x}_{1}, \boldsymbol{x}_{2}$-plane is considered. As depicted in Figure 9 the deformation phase is divided into steps. Up to a deformation of $e_{12} \leq 0.45$ only one contact zone in the RVE appears between the fibre bow and the lower fibre (see Figure 9(a)). For a deformation $e_{12}>0.45$ a second contact zone appears between the bow and the upper fibre; see Figure 9(b). During this 


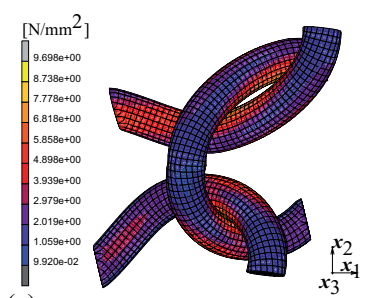

(a)

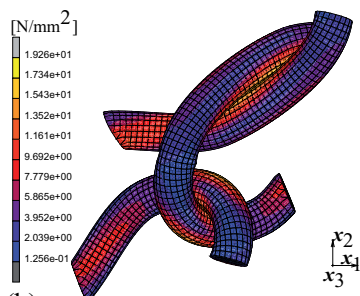

(b)

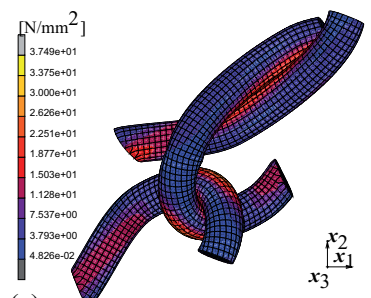

(c)

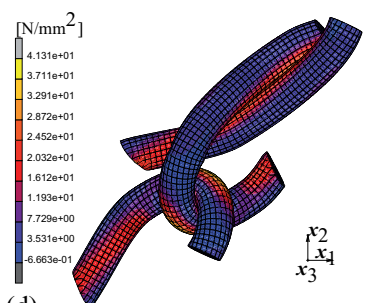

(d)

Figure 9. Von Mises plot of the representative volume element for shear deformation for $e_{12}$ of (a) 0.25 , (b) 0.45 , (c) 0.57 , (d) 0.70 .

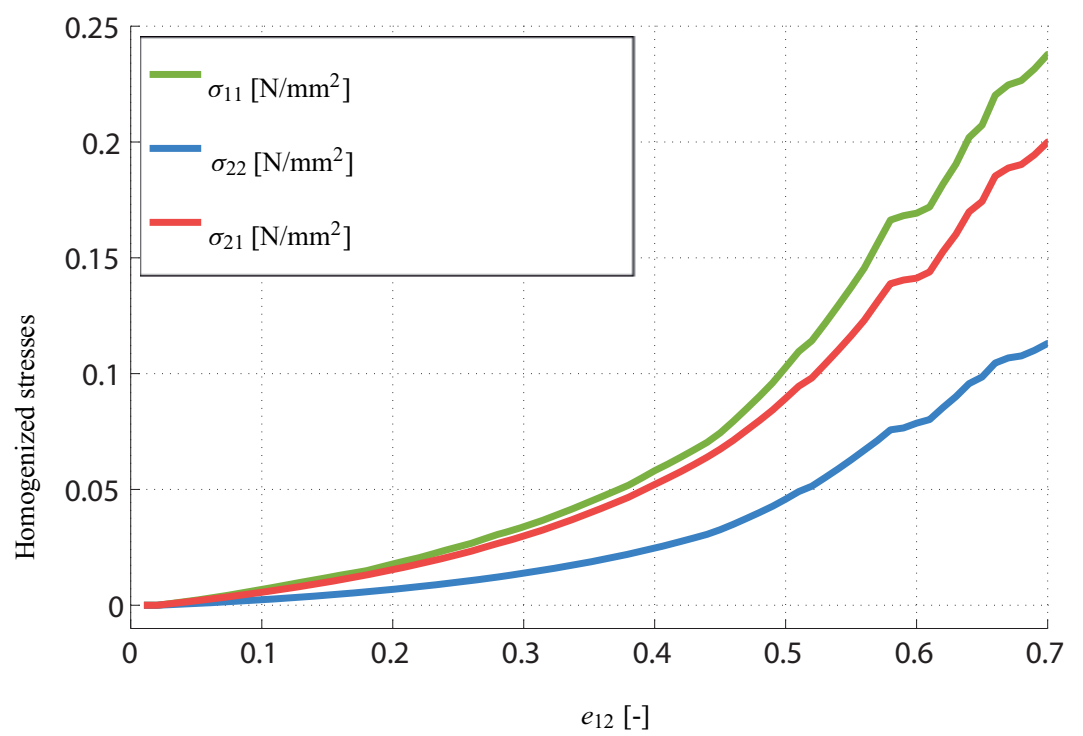

Figure 10. Macroscopic homogenized response of the knitted representative volume element for shear deformation.

deformation process the bow and the lower fibre turn out of the textile plane (Figure 9(c)) and the fibres in the other contact zone get more and more parallel (Figure 9(d)). Hence the fibres slide on each other. This behaviour of the structure can be also considered in the characteristic of the stresses, shown in Figure 10. Up to a deformation of about $e_{12}=0.45$ the homogenized stresses show a slight progression due to the increasing first contact zone. Thereafter, a second contact zone arises, which makes the RVE behaviour much stiffer. The slip between the nearly parallel lying fibres can be observed in the non-smoothness of the curve. The orthotropic material behaviour in the textile plane can be identified by the inequality of the stresses $\sigma_{11} \neq \sigma_{22}$ for shear deformation. Further, the knitted material is very flexible especially for shear deformation and shows a strong non-linear behaviour.

\section{Conclusions}

A composite material with periodic microstructure and multiple frictional micro-contact between structural elements was studied in the paper. The contact linear elasticity problem was considered in a multi-scale domain. Since the direct numerical computations on the fully resolved heterogeneous micro-structure are too expensive, a two-scale approach was used to obtain the effective mechanical behaviour of the material.

The recently obtained (see [1]) two-scale convergence result for the micro-contact elasticity problem was used for the statement of the auxiliary contact problem (9) in a periodicity cell or RVE and the homogenized stress-strain relation (10) was computed using this corrector problem. The limiting problem (11) can be associated with some elasto-plasticity: the frictional micro-sliding on the contact interface results in some irreversible deformations, which can be interpreted as plastic.

The algorithm was illustrated by its application to technical textiles. The effective in-plane behaviour of typical woven and knitted materials with periodic microstructure was computed and analysed. The macroscopic 
stress-strain relation is in general non-linear, in line with the theoretical expectations. Furthermore, the computations show that the macroscopic shear stress and stiffness are more sensitive to contact sliding between fibres than the macroscopic tension. While the macroscopic tensile stress-strain relation just becomes non-linear due to the presence of the contact, the macroscopic shear stiffness reduces significantly. However, this fact seems to be consistent with everyday practical experience with textile materials.

The non-linearity of the quasi-effective behaviour is furthermore revealed in unloading. The microscopic sliding results macroscopically in an irreversible deformation, which would result into hysteretic behaviour upon loading and unloading. This will be studied in detail in further publications.

\section{Conflict of interest}

None declared.

\section{Funding}

This research project is a cooperation of the projects STE 544/40-1 and OR 190/4-1 supported by the German Research Foundation (DFG).

\section{References}

[1] Damlamian, A, Cioranescu, D and Orlik J. Homogenization via unfolding in periodic elasticity with contact on closed and open cracks. Asympt Anal 2013; 82(3-4): 201-232.

[2] Kikuchi, N and Oden, JT. Contact problems in elasticity: A study of variational inequalities and finite element methods. Philadelphia, PA: SIAM, 1988.

[3] Eck, C and Jarusek, J. Existence results for the static contact problem with Coulomb friction. Math Mod Meth Appl S 1998; 8(3): 445-468.

[4] Bare, Z and Orlik, J. Asymptotics for a thin elastic fiber in contact with a rigid foundation. Proc Appl Math Mech 2011; 11(1): 501-502.

[5] Hummel, HK. Homogenization for heat transfer in polycrystals with interfacial resistances. Appl Anal 2000; 75: $403-424$.

[6] Yosifian, GA. On some homogenization problems in perforated domains with nonlinear boundary conditions. Appl Anal 1997; 65(3-4): 257-288.

[7] Yosifian, GA. Some homogenization problems for the system of elasticity with nonlinear boundary conditions in perforated domains. Appl Anal 1999; 71(1-4): 379-411.

[8] Mikelić, A, Shillor, M and Tapiéro, R. Homogenization of an elastic material with inclusions in frictionless contact. Math Comput Modell 1998; 28(4-8): 287-307.

[9] Sanchez-Palencia, E. Non-homogeneous media and vibration theory. Berlin/Heidelberg/New York: Springer-Verlag, 1980.

[10] Wriggers, P, Hain, M, Wellmann, C, et al. Homogenization of heterogeneous and granular material using finite and discrete elements. In: Proceedings of the international conference on computational plasticity COMPLAS IX, Barcelona, Spain, 2007, pp. $37-41$.

[11] Charmetant, A, Vidal-Salle, E and Boisse, P. Hyperelastic modelling for mesoscopic analyses of composite reinforcements. Compos Sci Technol 2011; 71: 1623-1631.

[12] Mielke, A and Rossi, R. Existence and uniqueness results for general rate-independent hysteresis problems. Math Models Methods Appl Sci 2007; 17: 81-123.

[13] Han, W. On the numerical approximation of a frictional contact problem with normal compliance. Numer Func Anal Optimiz 1996; 17(3-4): 307-321.

[14] Duvaut, G and Lions, JL. Inequalities in mechanics and physics. Berlin: Springer-Verlag, 1976.

[15] Cioranescu, D, Damlamian, A and Griso, G. Periodic unfolding and homogenization. C R Acad Sci Paris, Ser I 2002; 335: 99-104.

[16] Damlamian, A. An elementary introduction to periodic unfolding, multi scale problems and asymptotic analysis. In: Damlamian A, Lukkassen D, Meidell A, et al. (eds) GAKUTO International Series: Mathematical Sciences and Applications, vol. 24, 2005, pp. 119-136. Tokyo Japan: Gakkotosho.

[17] Panasenko, GP. Multi-scale modeling for structures and composites. New York, NY: Springer, 2005. 